\title{
Preface: Heart Valves
}

Reference to this organ, which perhaps was not commonplace in the scientific literature 15 years ago, has become exceedingly prevalent in the past 5 years. The field of heart valve research has become contemporary due to a heightened awareness of the seriousness and frequency of heart valve disease. Indeed, valve therapies are still extremely limited for patients above the age of 75 or less than 18; for this reason, several studies have been conducted recently in percutaneous valve therapies and in heart valve tissue engineering. When these studies are added to the body of ongoing research in pharmaceutical interventions, novel valve prosthetic materials, and the underlying mechanisms that lead to valve disease, several of which are only now being understood, it is clear that heart valve research has intensified to an unprecedented level. This special issue provides a glimpse into current areas of heart valve research, thereby reinforcing the interdisciplinary aspect of the work. Molecular biology, mechanics, materials, and regenerative medicine are some of the core subjects that comprise heart valve research today and are presented in this issue.

Mechanobiological aspects of heart valves are covered in the first third of this special issue. Cirka et al. start us off with insights into the well-known aortic valve interstitial cell. At the other end of the spectrum, Carneiro et al. present interesting work on a lesser-known population of melanocytes important in post-natal, murine heart valve development. These studies illustrate the heterogeneity of cellular populations that reside within the valve extracellular matrix as well as the complex interplay between mechanical environments and biological responses of these cell types. Next, Lam and Balachandran review the role of hemodynamics in drug-induced valvulopathies, focusing on serotonin-mediated disease.

The intermediate portion of this issue focuses on experimental and computational heart valve mechanics. Brazile et al. have examined bending properties of the four mammalian heart valves. Subsequently, computational modeling approaches elucidate the fatigue responses of collagenous valvular tissues (Martin \& Sun) and the flow physics past prosthetic heart valves resulting from fluid-structure interactions (Borazjani). Meanwhile, the latter part of this issue examines the utility of novel materials such as graphene (Lordeus et al.) and hydrogels (Zhang et al.) for developing the next generation of valve substitutes. In addition, Bhattacharya and $\mathrm{He}$ delineate the mechanics that result in repair procedures, specifically in the context of the mitral heart valve. Aptly, this series concludes with future regenerative strategies for promoting a valvular phenotype from a stem cell, mechanobiological perspective (Rath et al.).

All of the works presented herein represent critical reviews and/or original research reports on their chosen topic in the heart valve field. Even though these topics represent but a fraction of those possible, they nonetheless serve to effectively reflect the sufficient diversity of investigations that can evolve from the study of heart valve disease. Thus, from this special issue, it is hoped that the reader will gain a deeper appreciation and understanding of the nature of active heart valve research being carried out today.

\section{Guest Editor}

Sharan Ramaswamy, PhD, FAHA

Assistant Professor, Department of Biomedical Engineering

Florida International University

Miami, Florida, USA 
